\title{
The Effect of Mixing and Dissipation on A Lock-Exchange Flow Producedin A Channel of Constant Cross-Section
}

\author{
Tjipto Prastowo* \\ Physics Department, Surabaya State University (Unesa) \\ Ketintang Campus, Surabaya 60231
}

\begin{abstract}
Laboratory lock-exchanges of two fluids of different densities are used to examine the effects of mixing and dissipation on a dense current produced in a simple channel of constant width and height. Turbulent, small-scale mixing, induced by the flow instability at the interfacial layer, was observed above the current head. The mixing is found to affect the flow dynamics, in that the depth of the current is measured to be $0.36-0.47 \mathrm{H}$, less than a half of the full water depth $\mathrm{H}$, defined as a theoretically predicted depth, and that the non-dimensional current speed is constant at 0.480 .02 , independent of all the external parameters. From these finding, we conclude that there is a loss of energy associated with turbulent dissipation and irreversible mixing and that bottom friction plays a minor role in the propagation speed of the current.
\end{abstract}

KEYWORDS: dense current, current depth, current speed

\section{INTRODUCTION}

Lock-release exchange flows, or usually referred to as gravity currents, can be generated in the environment and in the laboratory when a fluid of one density propagates into a fluid of another density [1], where the difference in the fluid densities drives a two-layer exchange, with the less dense fluid overlying the denser fluid. [2] proposed a theory for propagating gravity currents and considered such currents as a twolayer flow. In this basic feature, the fluids were immiscible and the flow was steady, inviscid and hydrostatic. These made energy preserved to be conserved, for which the dense (occupying lower layer) current is theoretically predicted to occupy a half depth. This theory was supported by the laboratory results of $[3,4]$.

Many have examined the characteristics of gravity current either by laboratory work $[5,6]$, with further theoretical development by [7], or by numerical simulations [8, 9]. In this study, we focus on examining the effects of mixing and dissipation on a propagating gravity current produced in a simple, laboratory channel of constant cross-section by quantifying the depth and speed of the current.

This paper is then structured as follows. The experimental methods, including apparatus, procedure and parameters, are described in $\S 2$. The experimental results are presented in $\S 3$, followed by discussion in $\S 4$. Conclusions are given in $\S 5$.

*E-MAIL: t_prastowo@yahoo.com

\section{METHODS}

\section{A. Apparatus and procedure}

We carried out gravity current experiments in a horizontal tank of $5.3 \mathrm{~m}$ long and $0.2 \mathrm{~m}$ wide (Figure $1 \mathrm{a}$ ). In each experiment, the tank was filled with freshwater to a fixed depth of $0.2 \mathrm{~m}$ and a full width of a sliding barrier was placed in the centre of the tank. A density difference across the barrier was obtained by adding a measured amount salt to the rightside reservoir. Both reservoirs were stirred thoroughly to be homogeneous.

An experiment was initiated by sliding the barrier out of the tank. Gravity current formed on each of the reservoirs with the dense current traveling along the rigid base and the less dense current counter-flowing near the surface (Figure 1b). The barrier was reinserted into the tank when the nose of the dense current had reached the left endwall. The shadowgraph technique was used to visualise the flow and the digital video camera and digital still cameras were used to record the experiment.

The initial and final free surface heights were accurately determined using the digital micrometer to within $0.01 \mathrm{~mm}$. The densities before and after each experiment were measured using the digital density meter to a precision of $0.0001 \mathrm{~g} / \mathrm{cm} 3$. These accurate measurements were necessary in order to minimise errors.

\section{B. Parameters}

In the experiments, the non-dimensional density difference $\Delta \rho / \rho_{2}=\left(\rho_{2}-\rho_{1}\right) / \rho_{2}$ was varied, lying in the range 0.01 $\leq \Delta \rho / \rho_{2} \leq 0.07$, where $\rho_{1}$ and $\rho_{2}$ are the densities of the 


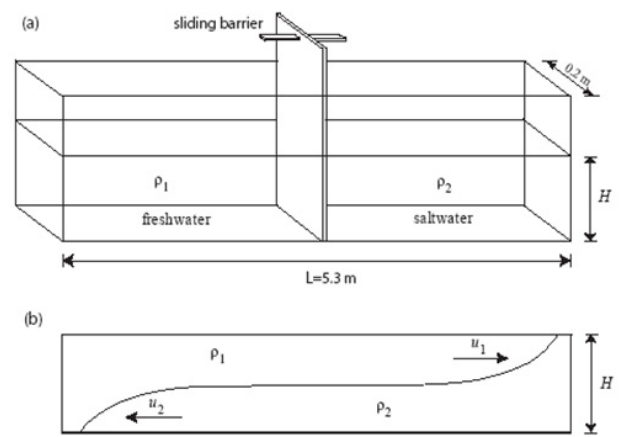

FIG. 1: (a) Laboratory apparatus used for experiments of lockrelease gravity currents and (b) a simplified feature of a propagating gravity current in the channel.

fresh (left-side) and saltwater (right-side) reservoirs, respectively. This fractional density difference is used to construct a vertical Reynolds number $R e_{H}=0.5\left(g^{\prime} H\right)^{1 / 2} / v$, where $\mathrm{g}^{\prime}=\mathrm{g} \Delta \rho$ is the reduced gravity, with $\mathrm{g}$ is acceleration due to gravity, $\mathrm{H}$ is the full water depth, and $\mathrm{v}$ is the kinematic viscosity. For the range of the fractional density differences used, this number is in the order of $10^{4}$.

\section{RESULTS}

\section{A. Flow features}

When the barrier was removed, the dense fluid collapsed and a characteristic head of a gravity current developed. As time progressed, gravity currents appeared as a bottom, dense current propagating along the channel base and a surface, less dense current overflowing in the opposite direction (Figure 1b). The development of the currents was the same for all experiments, in the sense that the shape of the head of the dense current was almost the same, independent of the density differences. The front shape was maintained when the dense current advanced steadily along the base. In all experiments, the bottom and surface currents were symmetric (Figure 1b).

The region above the head was unstable that led to billow formation and overturning, hence turbulent mixing between the fluid within the current and that of the surroundings. The mixing was observed to occur when the billows grew relatively large in size and collapsed, carrying away the mixed fluid from the head that was continually replaced by the fluid from the rear of the current. Thus, the sharp shape of the front could be sustained at a fixed shape and the corresponding speed was nearly constant. As the dense current moved forward, interfacial mixing also took place in the upper surface of the following current though it was less intense than that in the region above the head.

\section{B. Flow dynamics}

The depth of a gravity current is difficult to measure as the region above the head and along the upper surface of the following current are unstable and turbulent. Here, the current depth is estimated from a series of photographs, taken at times when the dense current have traveled away from the centre (Figure 2). The current depth is found to be $0.38-0.47 \mathrm{H}$, where $\mathrm{H}$ is the full depth, independent of positions at which photographs were taken.

The propagation speed of a gravity current is relatively easy to evaluate by examining the photographs of the dense current (Figure 2). The figure shows that the dimensionless front speed is constant at $0.48 \pm 0.02$, independent of all the parameters used. The uncertainty quoted here is due to the uncertainties in the measurements of the distance over which the front propagates and the time it takes for the front to travel such a distance.

\section{DISCUSSION}

The depth and speed of gravity current generated by lockexchange experiments are examined in relation to the effects of mixing and dissipation upon the flow dynamics. As with previous studies [5, 6], the current depth is relatively difficult to quantify as the region above and behind the head is highly unsteady and turbulent. However, the depth can be best estimated from the photographic and video records [4], with a better measurement of the current depth was proposed by [7] who found considerable variations in the current depth, from $0.35 \mathrm{H}$ to $0.50 \mathrm{H}$ for the range of the parameters used and initial conditions applied.

Using direct numerical simulations, [8] estimated the depth by vertically integrating the non-dimensional density profile of the flow field at a particular location and normalising it with the total depth. Using this, the depth was numerically estimated to be $0.30-0.40 \mathrm{H}$. This estimate shows a fundamental difference on the basis of a physical ground, as current heights much less than half depth indicate a loss of energy owing to viscous dissipation.

In the present experiments, the depth of the current is found between 0.38 and $0.47 \mathrm{H}$, consistent with the results of either laboratory measurements [7] or numerical simulations [8]. The present results suggest that the method for determining the current depth developed here is reliable.

The dimensionless current speed reported, $0.48 \pm 0.02$, agrees very well with the results of previous investigations $[6$, 7] and is very close to the theoretical non-dimensional speed of 0.5 for steady, non-dissipative gravity currents [2]. The experimental conditions considered in the current experiments, however, are different from those of [2], owing to the presence of mixing and friction.

From the measurements of the speed of the dense current, we argue that the nearly constant dimensionless speed indicates independence of the propagation speed of the current upon the mixing processes behind the current head and along the upper surface of the trailing current. As the small-scale 


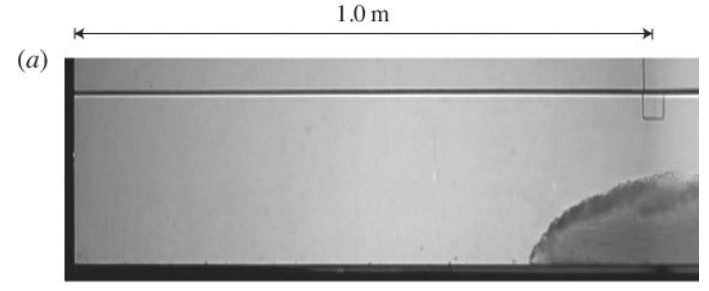

(b)

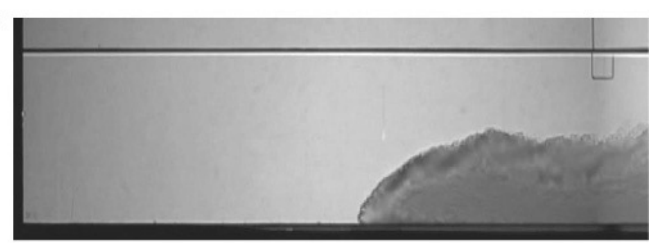

(c)

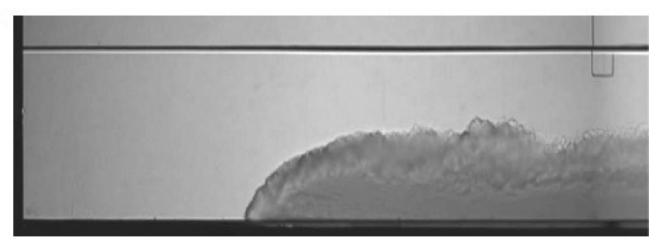

(d)

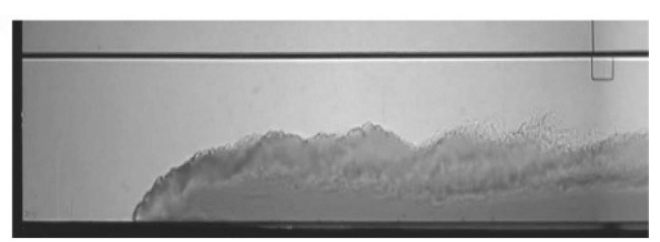

(e)

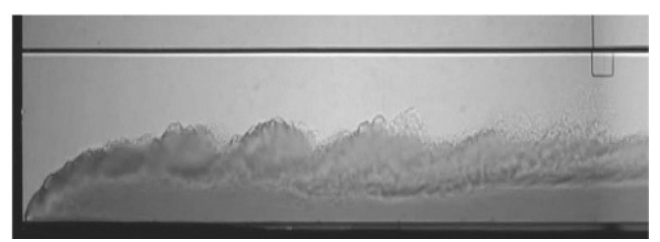

FIG. 2: Propagating dense current, with the total time taken for the front to propagate from frame (a) to frame (e) was $6.70 \mathrm{~s}$, where the distance between two consecutive frames was $0.20 \mathrm{~m}$. With g'= $0.29 \mathrm{~ms}^{-2}$ and $\mathrm{H}=0.20 \mathrm{~m}$, these photos suggest that the front traveled at a constant speed of $0.5\left(\mathrm{~g}^{\prime} \mathrm{H}\right)^{1 / 2}$. turbulence is always present in the experiments, there must be some loss of energy due to turbulent dissipation. Hence, the front speed is not a suitable measure of whether energy is conserved. Instead, the measurements of the current depth suggest that loss of energy, through flow processes associated with viscous dissipation and vertical redistribution of fluid density after mixing, cannot be fully negligible.

\section{CONCLUSIONS}

Laboratory measurements are performed to examine the effects of turbulent mixing and dissipation on gravity currents generated in a simple channel of constant width and depth. The measurements are used to determine the depth and speed of the current. The current depth is estimated to be between $0.36-0.47 \mathrm{H}$, in good agreement with laboratory investigations [7] and numerical simulations [8]. The current depth measured implies that some of the available potential energy is lost through irreversible mixing and the remaining potential energy is viscously dissipated. The dimensionless speed is measured to be $0.48 \pm 0.02$, independent of all the parameters used in the experiments and processes responsible for turbulent mixing. This speed also indicates that friction plays no role while the current propagating.

\section{Acknowledgments}

TP acknowledges the Australian Government for the award of ADS and would like to thank Prof. Ross Griffiths, Dr. Graham Hughes and Dr. Andy Hogg for their best help and kindness. Tony Beasley is also appreciated for technical assistance in the laboratory.
[1] Simpson, J. E., Gravity currents in the environment and the laboratory (2nd ed. Cambridge University Press. Cambridge, UK, 1997)

[2] Benjamin, T. B., J. Fluid Mech., 31, 209-248 (1968).

[3] Britter, R. E. and J. E. Simpson, J. Fluid Mech., 112, 459-466 (1981).

[4] Lowe, R. J., P. F. Linden, and J. W. Rotman, J. Fluid Mech., 456, 33-48 (2002).

[5] Garcia, M. H. and J. D. Parsons,Dyn. Atmos. Oce., 24, 197-205 (1996).
[6] Hacker, J., P. F. Linden, and S. B. Dalziel, Dyn. Atmos. Oce. 24, 183-195 (1996).

[7] Shin, J. O., S. B. Dalziel, and P. F. Linden, J. Fluid Mech., 521, 1-34 (2004).

[8] Hrtel, C., E. Meiburg, and F. Necker, J. Fluid Mech. 418, 189212 (2000).

[9] Necker, F., C. Hrtel, L. Kleiser, and E. Meiburg, J. Fluid Mech. 545, 339-372 (2005). 